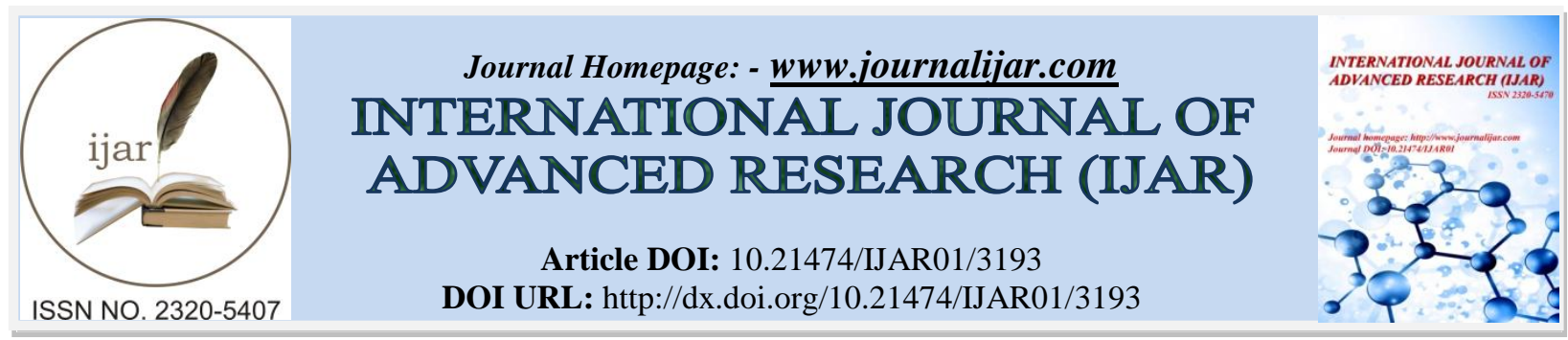

RESEARCH ARTICLE

\title{
PREVENTING DISABILITY AND MORTALITY RELATED TODIABETES IN THE PRIMARY CARE SETTING.
}

\section{Abdullatif Mohammed ALomair, Anas Abdulrahman Alarfaj, Marwan Mohammed ALothman, Abdullah saud ALotaibi, Mohammed Munif ALharbi, Abdulrahim Abdullatif ALomair and Abdullah Abdulaziz ALshuthri.}

Medical intern, King Faisal University Al Hasa, Saudi Arabia.

\section{Manuscript Info \\ Manuscript History \\ Received: 17 December 2016 \\ Final Accepted: 05 January 2017 \\ Published: February 2017}

Key words:-

Diabetes, Saudi Arabia, Prevention

\begin{abstract}
Diabetes mellitus is a leading cause of disability and death world-wide. However, many governments and public health planners remain largely unaware of the current prevalence of diabetes and prediabetes, the potential for a future rise in prevalence and the serious complications associated with this disease.
\end{abstract}

Copy Right, IJAR, 2017,. All rights reserved.

\section{Introduction:-}

Diabetes mellitus is a leading cause of disability and death world-wide. However, many governments and public health planners remain largely unaware of the current prevalence of diabetes and prediabetes, the potential for a future rise in prevalence and the serious complications associated with this disease. knowledge of the prevalence of diabetes and prediabetes related to risk factors could raise awareness of the disease and lead to new policy managements. There is a now abundant evidence that type 2 diabetes, which counts for greater than $90 \%$ of diabetes world-wide, is preventable ,therefore, Primary Health Care (PHC) is taken into account to be a good strategy to boost accessibility and usage of services, address the social determinants of health inequities and improve health outcomes through the implementation of comprehensive interventions through sectoral and intersectoral collaboration, direction of people, community participation and social mobilization. This study was to the contribution extent of primary health care in preventing diabetes prevalence. Therefore, this article proposes a study that will relay on the descriptive and analytical approach, also will be viewing on the literature and previous studies related to the subject of study. Questionnaires will be conducted among Health workers (Doctors, Nurses and Pharmacists) in Saudi Arabia to collect data on the causes of diabetes Prevalence, and the factors that lead to diabetes prevalence, goal setting; focus on Investigating the contribution of primary health care in preventing diabetes prevalence and examine whether the relationship between primary health care and the spread of diabetes.

\section{Methods:-}

This study depends on the descriptive and analytical approach, also will be viewing on the literature and previous studies related to the subject of study. For examining the contribution of primary health care in preventing diabetes prevalence

For this purpose the researcher will distribute questionnaires for preliminary information for analysis. The study will be conducted among Health workers (Doctors, Nurses and Pharmacists) in al Alaha hospitalsSaudi Arabia. 
Questionnaires will delivered to Doctors, Nurses and Pharmacists working in health sector to collect data on the causes of diabetes injury, and the factors that lead to diabetes prevalence. Statistical methods will be used by the statistical program for Social Sciences (SPSS) to handle the data obtained.

\section{Results:-}

Diabetes is a chronic condition related to multiple late complications, reduced life, and a marked limitation within the quality of life. The malady, its complications and late-onset consequences cause a dramatic burden for health systems.

Primary Health Care (PHC) is taken into account to be a good strategy to boost accessibility and usage of services, address the social determinants of health inequities and improve health outcomes through the implementation of comprehensive interventions through sectoral and intersectoral collaboration, direction of people, community participation and social mobilisation.

This study was to the contribution extent of primary health care in preventing diabetes prevalence.

\section{Discussion:-}

Stellefson et al (2013) created a study that looked at the Chronic Care Model and Diabetes Management in US Primary Care Settings: A Systematic Review, The Chronic Care Model (CCM) uses a systematic approach to restructuring medical care to create partnerships between health systems and communities. The objective of this study was to describe how researchers have applied CCM in US primary care settings to provide care for people who have diabetes and to describe outcomes of CCM implementation. To achieve the goal of the study, the researchers conducted a literature review by using the Cochrane database of systematic reviews, CINAHL, and Health Source: Nursing/Academic Edition and the following search terms: "chronic care model" (and) "diabet*.", also included articles published between January 1999 and October 2011. We summarized details on CCM application and health outcomes for 16 studies, The 16 studies included various study designs, including 9 randomized controlled trials, and settings, including academic-affiliated primary care practices and private practices. Results of the study showed that CCM approaches have been effective in managing diabetes in US primary care settings. Organizational leaders in health care systems initiated system-level reorganizations that improved the coordination of diabetes care. Disease registries and electronic medical records were used to establish patientcentered goals, monitor patient progress, and identify lapses in care. Primary care physicians (PCPs) were trained to deliver evidence-based care, and PCP office-based diabetes self-management education improved patient outcomes. Only 7 studies described strategies for addressing community resources and policies. The researchers recommended that future research about integration of CCM into primary care settings for diabetes management should measure diabetes process indicators, such as self-efficacy for disease management and clinical decision making.

Additionally, Green et al (2012)also created a study about" Primary prevention of type 2 diabetes: integrative public health and primary care opportunities, challenges and strategies", Type 2 diabetes imposes a large and growing burden on the public's health. This burden, combined with the growing evidence for primary prevention from randomized controlled trials of structured lifestyle programs leads to recommendations to include caloric reduction, increased physical activity and specific assistance to patients in problemsolving to achievemodest weight loss as well as pharmacotherapy. These recommendations demand exploration of new ways to implement such primary prevention strategies through more integrated community organization, medical practice and policy. The US experience with control of tobacco use and high blood pressure offers valuable lessons for policy, such as taxation on products, and for practice in a variety of settings, such as coordination of referrals for lifestyle supports. We acknowledge also some notable exceptions to their generalizability. This paper presents possible actions proposed by an expert panel, summarized in Table 1 as recommendations for immediate action, strategic action and research. The collaboration of primary care and public health systems will be required to make many of these recommendations a reality. This paper also provides information on the progressmade in recent years by the Division of Diabetes Translation at the US Centers for Disease Control and Prevention (CDC) to implement or facilitate such integration of primary care and public health for primary prevention.

Furthermore, Harris(2008), titled "The role of primary health care in preventing the onset of chronic disease, with a particular focus on the lifestyle risk factors of obesity, tobacco and alcohol", the potential impact of primary health care in assessing and managing smoking, hazardous drinking, poor diet and physical inactivity has been 
demonstrated among patients who are at higher risk. Effective interventions based around the 5As approach include the assessment of risk and readiness to change, brief motivational interventions and referral of suitable patients for more intensive interventions. Health checks have been demonstrated to improve the frequency of preventive care and support for behavior change. However their impact on health outcomes is uncertain. Expansion of the current complexity of different Medicare items is undesirable. Health checks should focus on specific evidence based preventive actions and involves the use standardized resources such as Life scripts and integrated health risk assessment tools. Other performance based incentives may be useful in targeting the needs of specific groups and encouraging primary health care to provide more outreach and culturally appropriate preventive care for disadvantaged groups. General practice referral of patients needing more intensive lifestyle interventions is infrequent due to a number of factors including cost and availability of providers and services and the integration between these services and primary health care. A major role of primary health care organisations is to coordinate and broker a network of referral services to support behavior change based on standards and quality assurance. Other key roles of primary care include the support of practices to monitor and improve their performance in providing preventive care. Structural reform of primary health care may provide opportunities for more integrated approaches to management of the lifestyle risk factors across private and public and national and state funded services.

\section{Conclusion:-}

Diabetes: is a serious, chronic disease that occurs either when the pancreas does not produce enough insulin (a hormone that regulates blood glucose), or when the body cannot effectively use the insulin it produces.

\section{References:-}

1. Akter, Shamima; Rahman, M Mizanur; Abe, Sarah Krull \& Sultana, Papia. 2014. Prevalence of diabetes and prediabetes and their risk factors among Bangladeshi adults: a nationwide survey, Bulletin of the World Health Organization, 92:204-213A, doi: http://dx.doi.org/10.2471/BLT.13.128371.

2. Campbell S, Steiner A, Robison J, Webb D, Raven A, Roland M. 2003. Is the quality of care in general medical practice improving? Results of a longitudinal observational study, Br J Gen Pract , 53:298-304

3. Erny-Albrecht, Katrina; Bywood, Petra \& Oliver-Baxter, Jodie.2015. The role of primary health care in primary and secondary prevention of diabetes, Primary Health Care Research \& Information Service (PHCRIS), ISBN 978-0-9941874-3-7.

4. Green,Lawrence W.; Brancati, Frederick L. \& Albright,Ann. 2012. Primary prevention of type 2 diabetes: integrative public health and primary care opportunities, challenges and strategies, Published by Oxford University Press, Family Practice 2012; 29:i13-i23. doi:10.1093/fampra/cmr126.

5. Harris, Mark. 2008. The role of primary health care in preventing the onset of chronic disease, with a particular focus on the lifestyle risk factors of obesity, tobacco and alcohol, Centre for Primary Health Care and Equity, UNSW.

6. Marcos, Cueto. 2004. The Origins of Primary Health Care and Selective Primary Health Care, Am J Public Health. 22. 94: 1864-1874. doi:10.2105/ajph.94.11.1864

7. McGlynn EA, Asch SM, Adams J, Keesey J, Hicks J, DeCristofaro A. 2003. The quality of health care delivered to adults in the United States, N Engl J Med, 348:2635-45.

8. Shi, Leiyu. 2012. The Impact of Primary Care: A Focused Review, Review Article, Volume, Article ID 432892, 22 pages, Johns Hopkins Bloomberg School of Public Health, 624 North Broadway, Baltimore, MD 21205, USA,http://dx.doi.org/10.6064/2012/432892.

9. Stellefson, Michael; Dipnarine, Krishna \& Stopka, Christine. 2013. The Chronic Care Model and Diabetes Management in US Primary Care Settings: A Systematic Review, centers for disease control and prevention, public health research, practice, and policy, Volume 10, Prev Chronic, DOI, http://dx.doi.org/10.5888/pcd10.120180.

10. Tarlier, DS.; Johnson, JL. \& Whyte, NB. 2003. Voices from the wilderness: an interpretive study describing the role and practice of outpost nurses, Canadian Journal of Public Health, 94(3), 180-184.

11. Thomas-MacLean, R.; D. Tarlier, M. Fortin, S. Ackroyd-Stolarz, M. Stewart. 2014. No Cookie-Cutter Response: Conceptualizing Primary Health Care, Retrieved 26 August 2014. 